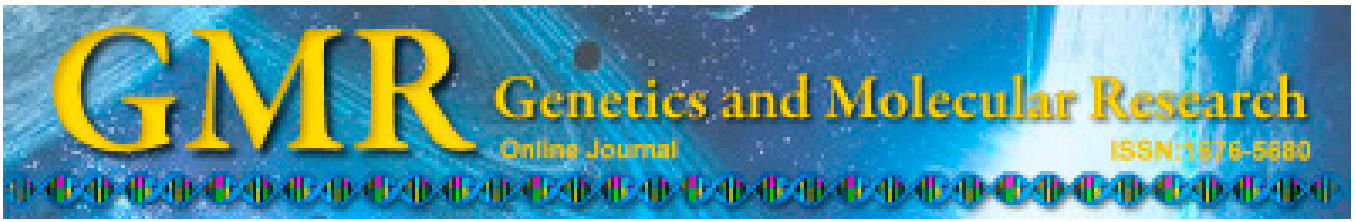

\title{
Analysis of the GSTM1-null polymorphism in patients with pterygium from Goiânia, Goiás Brazil
}

\author{
A. de P.R. Júnior ${ }^{1}$, G.M. dos Reis ${ }^{1}$, K.S.F. e Silva ${ }^{2}$, D.A. Rodrigues ${ }^{1}$, \\ M.C.S. Gomes ${ }^{1}$, J.V.M. Martins ${ }^{1}$, I.R. da Costa ${ }^{1}$, G.A. Freitas ${ }^{1}$ and \\ K.K.V.O. Moura ${ }^{2}$ \\ ${ }^{1}$ Replicon, Pontifícia Universidade Católica de Goiás, Goiânia, GO, Brasil \\ ${ }^{2}$ Laboratório de Genética e Biologia Molecular, \\ Universidade Federal de Goiás, Goiânia, GO, Brasil \\ Corresponding author: K.S.F. e Silva \\ E-mail: smallbinho@hotmail.com
}

Genet. Mol. Res. 14 (2): 6173-6181 (2015)

Received August 12, 2014

Accepted March 6, 2015

Published June 9, 2015

DOI http://dx.doi.org/10.4238/2015.June.9.3

\begin{abstract}
The first reports about pterygium date back to Hippocrates, and this disease still threatens vision health around the world. Pterygium is a formation of fibrous tissue consisting of highly vascularized epithelial and subepithelial tissue that grows excessively and with an abnormal shape on the cornea. Many physical and biological factors are associated with the pathogenesis of pterygium, including heat, dust, and other particles in the atmosphere, and immunological mechanisms, mechanisms involving extracellular matrix reorganization, growth factors, cytokines, apoptosis, and angiogenesis. The aim of this study was to further investigate the association between polymorphisms in GSTM1 and the formation of pterygium. We collected peripheral blood samples from 90 patients diagnosed with pterygium and from 23 subjects without the disease in order to perform molecular analysis of the GSTMI gene. Subjects with one or two copies of the GSTM1 allele had a normal genotype while those without any copies of the allele had a null genotype. The chi-square test or the Fisher exact test was performed in order
\end{abstract}


to investigate possible associations between the molecular analysis and the risk of pterygium. A significant difference between the frequency of the GSTM1-null genotype in patient and control groups was identified. However, sub-group analysis found that the GSTM1-null genotype was statistically significant in men, but not in women, and in Caucasians, but not in Brown or Black groups. Furthermore, the GSTM1-null genotype was not related to any of the risk factors analyzed: cases in family, occupational exposure, smoking, hypertension, and diabetes.

Key words: GST; GSTM1; Pterygium; Polymorphism

\section{INTRODUCTION}

Even though the first reports about pterygium date back to Hippocrates, its pathogenesis is not well-understood and it continues to threaten the vision health of many people around the world. The current description of the disease is similar to those from the past: a fibrous tissue mass is formed and moves across the surface of the cornea, assuming a triangular shape (Di Girolamo et al., 2004).

Pterygium is one of the most common known eye diseases. It is formed by fibrous connective tissue that is highly vascularized and grows abnormally on the cornea. Although pterygium can cause eye irritation in any phase of the disease, it is usually painless. Symptoms are related to foreign body and ocular burning sensations and red eyes. Signs of moderate to chronic conjunctivitis and thickening of the conjunctiva are also frequent and recurring symptoms. Diagnosis is performed by physical examination and is supplemented by biomicroscopy (Di Girolamo et al., 2004).

Pterygium is a benign condition of high worldwide prevalence and its high recurrence rate makes surgical procedures necessary (Golu et al., 2011). Countries located in the tropical zone show a pterygium prevalence of $22 \%$ while countries outside that area show a prevalence of $2 \%$ (Shiroma et al., 2009).

There is a higher incidence of pterygium in men, and this can be explained by different lifestyles between men and women: men generally spend more time outdoors than do women. Working outdoors, sun exposure, and other environmental factors such as dust can lead to chronic eye irritation and influence the onset of pterygium (Di Girolamo et al., 2004). Overall, the high correlation of the disease with ultraviolet (UV) radiation confirms its influence on the pathology of the disease. The anatomical structures around the eye (orbital rim, eyebrow, and nasal prominence) guarantee a degree of protection against the direct effects of UV rays. Nevertheless, protection is compromised when the exposure is through the bottom of the eye orbit or laterally (Golu et al., 2011). The negative effects of UV rays on the eyes are cumulative, and intense UV exposure means greater risk. Therefore, the use of sunglasses for eye protection is an important prophylactic measure, and it is important that sunglasses are used throughout the year and not only in the summer (Tung et al., 2010). The use of special lenses that filter UV rays with high efficiency is important when sun exposure is necessary and inevitable (Chui et al., 2008). Optical-quality sunglasses are able to block $100 \%$ of UV-A and UV-B radiation if they filter from 75 to $90 \%$ of the visible light (Said et al., 2007). Accessories such as hats and visors, among others may offer additional protection, especially if there is a need for prolonged sun exposure (Di Girolamo et al., 2004). 
Another cause of degenerative diseases of the eye such as pterygium is oxidative stress. Oxidative stress is understood as an imbalance between the formation and removal of oxidizing agents in a tissue, caused by excessive production of reactive oxygen species or by a decrease in endogenous antioxidants. Free radicals, such as superoxide anion, hydroxyl, alkoxy, peroxyl, and hydroperoxyl groups are chemical species that contain unpaired electrons in their outer electron orbitals, whereas reactive oxygen species are any oxidizing compounds (Evereklioğlu et al., 2003).

Reactive oxygen species are formed in several different situations in an organism: for example, in the production and regulation of nucleic acids, the detoxification of xenobiotics, during the actions of immune system cells following an inflammatory response, in transducing cellular signals, and resulting from environmental influence (exposure to UV radiation). One of the most important mechanisms of production of reactive oxygen species is energy metabolism. The excess of reactive compounds causes damage to many tissues through their reaction with cellular structures, contributing to aging and chronic and autoimmune diseases (Solomon, 2006).

Glutathione S-transferases (GSTs) belong to a family of multifactorial enzymes with the function of catalysis of the nucleophilic attack of glutathione in its reduced form (GSH) (Hayes et al., 2005). GSTs are one of the enzyme types responsible for protection against the oxidation caused by reactive oxygen species. In humans, the GST family can be divided into the classes $\alpha, \mu, \pi, \sigma, \kappa, \varepsilon$, and $\theta$. The $\mu$ family locus, commonly called (M), is located on chromosome 1 (1p13.3). There are three alleles: GSTM1 (0), GSTM1 (A), and GSTM1 (B). GSTM1 (0) corresponds to a deletion and the homozygote expresses neither the mRNA nor the corresponding protein. The GSTM1 (A) and (B) alleles encode monomers that form homo- and heterodimeric enzymes. GSTM1 0/0 is usually called the GSTM-null polymorphism (Tsai et al., 2004).

Classes $\mu$ (GSTM1) and $\theta$ (GSTT1) are polymorphic genes and, according to Scianni et al. (2008), they are homozygous in about 50 and $24 \%$ of the different ethnic populations, respectively. The null genotype is a consequence of unequal recombination. The deletion of $15 \mathrm{~kb}$ contains the entire gene (Hayes et al., 2005). Some authors have suggested that human GSTT1 and GSTM1 take part in the deactivation of reactive oxygen species that would otherwise be involved in the cellular processes of inflammation, aging, and degenerative diseases (Bolth and Thier, 2006).

Tsai et al. (2004) demonstrated that the GSTM1-null genotype was associated with an increased susceptibility to early onset of pterygium, although it was not associated with lateonset pterygium cases. The authors suggested that GSTM1 might be an important antioxidant involved in the metabolism of xenobiotics (Young et al., 2010).

It has been proposed that cumulative DNA damage might contribute to the development of certain diseases. For example, patients with DNA repair deficiencies are more likely to develop neoplastic diseases. Deletion of GSTM1, which characterizes the null genotype, is associated with the absence of enzymatic function and an increase in cytogenetic damage (Tsai et al., 2004). The GSTM1-null genotype has also been associated with an increased risk of developing pterygium. The expression of GSTM1 is thought to be important in protecting corneal cells against reactive oxidants, as these cells exhibit marked photosensitivity. Subjects with the GSTM1-null genotype were reported to show more intense inflammatory reactions after exposure to UV radiation, and they had a higher prevalence of skin cancer (Tung et al., 2010). Such genetic studies are an important step in understanding the pathogenesis of pterygium. Genetic markers can be used to identify patients at risk and to develop preventive measures for those patients that could reduce the likelihood of developing pterygium (Weiss 
et al., 2005). Accordingly, the aim of the present study was to analyze the relationship between the GSTM1-null polymorphism and pterygium.

\section{MATERIAL AND METHODS}

All subjects that took part in the present study answered a social and economic questionnaire containing basic information, including name, ethnicity, and drinking alcohol consumption and/or smoking habits. All patients signed an informed consent form. We collected peripheral blood samples from 90 patients diagnosed with pterygium, and from 23 subjects free from the disease to form the control group. Blood samples were collected at the Hospital Banco de Olhos in the city of Goiânia, Goiás. The molecular analysis to determine the GSTMI genotype was performed at Replicon-PUC-Goiás.

Genomic DNA was extracted and purified using the Illustra ${ }^{\mathrm{TM}}$ blood genomic Prep Mini Spin Kit (GE Healthcare ${ }^{\circledR}$, USA). The DNA samples were quantified using a NanoVue ${ }^{\mathrm{TM}}$ Plus (GE Healthcare) according to manufacturer instructions. DNA samples that were at a minimal concentration of $5 \mathrm{ng} / \mu \mathrm{L}$ were used in the analysis.

After extraction, the DNA samples were subjected to amplification by polymerase chain reaction (PCR) to detect allele-specific polymorphism of the GSTT1, according to the protocol of Frare et al. (2013). PCR products were subjected to electrophoresis on a $2 \%$ agarose gel with an electric field of $10 \mathrm{~V} / \mathrm{cm}$, and then stained with ethidium bromide $(5 \mathrm{mg} / \mathrm{mL})$. The gel was visualized and documented by UV transillumination on an ImageMaster ${ }^{\mathrm{TM}}$ VDS $^{\circledR}$ (Amersham Pharmacia Biotech, GE Healthcare, Munich, Germany). Subjects with one or two copies of the GSTT1 allele have a normal genotype while those without any copies of the allele have a null genotype. The number of alleles was determined by electrophoresis gel (Frare et al., 2013). Genotypes were recorded in spreadsheets using the Microsoft Excel ${ }^{\circledR} 2003$ software (Microsoft Corp., Redmond, WA, USA). Subsequently, the chi-square test or the Fisher exact test was performed in order to investigate possible associations between molecular analysis results and the incidence of pterygium. We used BioEstat ${ }^{\mathbb{B}}$ 3.0 (Mamirauá/MCT-CNPq Civil Society, Belém, Brazil) to perform the analysis.

\section{RESULTS}

The frequencies of the GSTM1 gene polymorphism were analyzed in the patient (case: patients with pterygium) and control (subjects free from the disease) groups as shown in Table 1. In the patient group, containing 90 individuals, the GSTM1 gene could be detected in $17.6 \%$ $(15 / 90)$ of the subjects, whereas $83.3 \%$ (75/90) carried the null polymorphic genotype. In the control group (containing 23 subjects) we detected $43.5 \%$ (10/23) positive for the GSTM1 gene and 56.5\% (13/23) with the null polymorphic genotype. The results show a statistically significant difference confirming a positive relationship between the GSTM1-null genotype and pterygium $(\mathrm{P}=0.0056)$.

Table 2 shows the genotype distribution of GSTM1 in relation to gender in the patient and control groups. Analysis of the GSTM1 polymorphism in women in the patient group detected 20\% (10/50) of the patients with the GSTM1-present genotype and 80\% (40/50) with the null polymorphic genotype. Of females in the control group, 33.3\% (4/12) had the GSTM1-present genotype and $66.7 \%(8 / 12)$ had the GSTM1-null genotype. This result was not statistically significant $(\mathrm{P}=0.2631)$. 
Analysis of the GSTMI polymorphism in men demonstrated that $12.5 \%(5 / 40)$ of the patient group had the GSTM1-present genotype, while $87.5 \%(35 / 40)$ had the null polymorphic genotype. In the control group, 54.5\% (6/11) of the men had the GSTM1-present genotype and $45.5 \%(5 / 11)$ had the null polymorphic genotype. The difference in this frequency was statistically significant $(\mathrm{P}=0.0071)$, which supports the hypotheses of a higher incidence of pterygium in men.

Table 1. Distribution of the GSTM1 polymorphism in patient and control groups.

\begin{tabular}{lccccc}
\hline & \multicolumn{2}{c}{ GSTM1-present } & \multicolumn{2}{c}{ GSTM1-null } & P \\
\cline { 2 - 5 } & $\mathrm{N}$ & $\%$ & $\mathrm{~N}$ & $\%$ & 0.0056 \\
\hline Patients $(\mathrm{N}=90)$ & 15 & 16.7 & 75 & 83.3 & \\
Controls $(\mathrm{N}=23)$ & 10 & 43.5 & 13 & 56.5 & \\
\hline
\end{tabular}

$\mathrm{P}$ value from the chi-square test.

Table 2. Distribution of the GSTM1 polymorphism in relation to gender in the patient and control groups.

\begin{tabular}{|c|c|c|c|c|c|c|c|}
\hline \multirow[t]{2}{*}{ Gender } & \multicolumn{2}{|c|}{ GSTM1-present } & \multicolumn{2}{|c|}{ GSTM1-null } & \multicolumn{2}{|c|}{ Total } & \multirow[t]{2}{*}{$\mathrm{P}$} \\
\hline & $\mathrm{N}$ & $\%$ & $\mathrm{~N}$ & $\%$ & $\mathrm{~N}$ & $\%$ & \\
\hline \multicolumn{8}{|l|}{ Female } \\
\hline Patients & 10 & 20.0 & 40 & 80.0 & 50 & 100.0 & 0.2631 \\
\hline Controls & 4 & 33.3 & 8 & 66.7 & 12 & 100.0 & \\
\hline \multicolumn{8}{|l|}{ Male } \\
\hline Patients & 5 & 12.5 & 35 & 87.5 & 40 & 100.0 & 0.0071 \\
\hline Controls & 6 & 54.5 & 5 & 45.5 & 11 & 100.0 & \\
\hline
\end{tabular}

$\mathrm{P}$ value from the Fisher exact test.

Table 3 shows the GSTM1genotype distribution among different ethnic groups: Caucasian, and patients with brown or black skin. Analysis of the GSTM1 gene in the Caucasian patients found that 12\% (6/50) had the GSTM1-present genotype and 88\% (44/50) had the GSTM1-null genotype; $50 \%(8 / 16)$ of subjects in the control group showed GSTM1present genotype and 50\% (8/16) showed the GSTM1-null genotype. This result was statistically significant $(\mathrm{P}=0.0030)$ and it supports the idea that the disease is more frequent in Caucasian patients.

Table 3. Comparison between skin color and the GSTM1 polymorphism in patient and control groups.

\begin{tabular}{|c|c|c|c|c|c|}
\hline \multirow[t]{2}{*}{ Groups } & \multicolumn{2}{|c|}{ Patients } & \multicolumn{2}{|c|}{ Controls } & \multirow[t]{2}{*}{$\mathrm{P}$} \\
\hline & $\overline{\mathrm{N}}$ & $\%$ & $\overline{\mathrm{N}}$ & $\%$ & \\
\hline \multicolumn{6}{|l|}{ *Caucasian } \\
\hline GSTM1-present & 6 & 12.0 & 8 & 50.0 & 0.0030 \\
\hline GSTM1-null & 44 & 88.0 & 8 & 50.0 & \\
\hline Total & 50 & 100.0 & 16 & 100.0 & \\
\hline \multicolumn{6}{|l|}{ *Brown skin } \\
\hline GSTM1-present & 5 & 21.7 & 1 & 33.3 & 0.5538 \\
\hline GSTM1-null & 18 & 78.3 & 2 & 66.7 & \\
\hline Total & 23 & 100.0 & 3 & 100.0 & \\
\hline \multicolumn{6}{|l|}{ *Black skin } \\
\hline GSTM1-present & 4 & 23.5 & 1 & 25.0 & 0.6683 \\
\hline GSTM1-null & 13 & 76.5 & 3 & 75.0 & \\
\hline Total & 17 & 100.0 & 4 & 100.0 & \\
\hline
\end{tabular}

P value from the Fisher exact test. *As reported by the patient. 
In patients with brown skin, $21.7 \%(5 / 23)$ of the subjects belonging to the patient group had the GSTM1-present genotype and $78.3 \%(18 / 23)$ had the polymorphic null genotype. In the control group, $33.3 \%(1 / 3)$ had the GSTM1-present genotype and $66.7 \%(2 / 3)$ had the GSTM1-null genotype. The results for this pseudo-ethnic group showed no statistically significant difference $(\mathrm{P}=0.5538)$.

The analysis of GSTM1 polymorphisms in patients with black skin found that $23.5 \%$ $(4 / 17)$ of the subjects in the patient group showed the normal genotype and 76.5\% (13/17) showed the null GSTM1 genotype. One quarter $(25 \% ; 1 / 4)$ of the subjects in the control group showed the normal genotype and $75 \%(3 / 4)$ showed the null polymorphic genotype. No statistically significant difference was identified between the patient and control groups $(\mathrm{P}=0.6683)$.

Furthermore, the GSTM1-null genotype was not found to be related to the other risk factors analyzed: cases in family, occupational exposure, smoking, drinking, hypertension, and diabetes.

\section{DISCUSSION}

Pterygium is a serious public health problem. It is often diagnosed at a routine eye exam and prevention campaigns against pterygium are rarely performed. Several studies have been conducted in order to identify a molecular genetic component of this disease (Jaworski et al., 2009). One candidate, the GSTM1 gene product, plays a key role in protecting tissues against oxidative stress that could lead to diseases such as pterygium (Tung et al., 2010).

Our analysis of GSTM1 polymorphic variants found a statistically significant difference between the GSTM1-null genotype in patient and control groups when the frequencies between these groups were compared. A separate study comprising 127 patients confirms our results; they found that the null genotype of the GSTM1 gene is associated with the onset of pterygium (Tsai et al., 2004). However, two independent studies in China (Young et al., 2010) and in Taiwan (Tung et al., 2010) analyzed the GSTM1-null polymorphism in patients with pterygium and found no significant differences. The discrepancy in the results might be explained by the populations used in these studies. The Brazilian population is characterized by miscegenation, and has ancestral roots in Europe, Africa, and America with various migration patterns (Suarez-Kurtz, et al., 2014). In China (Young et al., 2010) and Taiwan (Tung et al., 2010), the populations are more homogeneous, having an Asian origin.

In our study, we found that the presence of the GSTM1-null genotype was related to the onset of pterygium in men. However, there was no significant difference in the prevalence of pterygium in women for this polymorphism. A study conducted in Australia (Sherwin et al., 2013) showed that the frequency of the GSTM1-null genotype is more frequent in the male population of that country, a result that corroborates the results in the present study. Other studies also corroborate our results.

A group of studies from Beijing (Ma et al., 2007) and from Ethiopia (Meseret et al., 2008) showed that pterygium is more frequent in men. However, a Chinese study analyzed the GSTM1-null polymorphism but found no significant difference between men and women (Young et al., 2010). As for the incidence of pterygium itself, the discrepancies in the published results might be explained by the different populations used in each study. Unlike the Brazilian population, the Australian population originates primarily from Europe (Sherwin et al., 2013), the population from Beijing has an Asian origin (Ma et al., 2007), and the population of Ethiopia is mostly Semitic (Meseret et al., 2008). 
A study conducted in Barbados (Luthra et al. 2001) also showed that ethnicity might be related to the prevalence of pterygium. They analyzed 4709 patients and found that the prevalence of pterygium in that country is related to the Black population. Pterygium is 2.5 to 3.0 times more frequent in the Black patients compared to the rest of the population (Luthra et al. 2001). In contrast, a group from Spain showed that the prevalence of pterygium was higher in Caucasians, which supports our results (Viso et al., 2011).

In the present study, we observed that the presence of the GSTM1-null genotype did not increase the possibility of developing the disease in patients who had ocular abnormalities in the family. Unlike our results, a study in Africa (Anguria et al., 2012) showed that cases of pterygium in the family can contribute to increasing the frequency of the disease in subsequent generations. Again, the discrepancy in results might be due to the different populations studied. The miscegenistic Brazilian population (Suarez-Kurtz et al., 2014) supports considerable genetic heterogeneity, whereas the African population studied was more homogeneous with respect to their genetic characteristics (Anguria et al., 2012).

We observed that the GSTM1-null genotype did not increase the possibility of developing pterygium when there was occupational exposure. A study in Malaysia (CajucomUy et al., 2010) supports our results. They found no significant differences when comparing pterygium and occupational exposure in that population. On the other hand, a research group from Barbados (Luthra et al., 2001) showed that pterygium was twice as frequent in patients who had occupational exposure. The authors claimed that it was very important to invest in prevention programs that encouraged people who work while exposed to the sun to use proper equipment in an attempt to decrease the chance of developing pterygium.

In this case, we believe that any discrepancies in results might be explained by the small sample size of this research, which represent a genotypic GSTM1 profile of the central Western Brazil. The present study has shown that the presence of the GSTM1-null genotype did not increase the possibility of developing pterygium in patients who smoke. These results are supported by those from a group of scientists from India (Asokan et al., 2012), who evaluated the effect of smoking in relation to pterygium. A further study conducted with Latin American patients living in the United States also found no significant difference regarding pterygium and smoking (West and Muñoz, 2009).

We found that the presence of the GSTM1-null genotype did not increase the possibility of developing pterygium when the patient suffered from hypertension. Similarly, a study conducted in China (Zhao et al., 2013) followed 4439 patients for 10 years, and found that there was no relationship between the onset of the disease and hypertension. We also found that the presence of the GSTM1-null genotype did not increase the risk of developing pterygium when the patient had diabetes, and, in addition, we demonstrated that pterygium was not related to alcohol consumption. A study group from China (Zhong et al., 2012) assessed several parameters regarding the onset and development of pterygium such as height, weight (not evaluated by our group), hypertension, drinking, smoking, and diabetes. Their results support our results: there was no statistical significance between pterygium and diabetes or alcohol consumption. The results overall, therefore, demonstrate that the GSTM1-null genotype is not related to the following risk factors: cases in family, occupational exposure, smoking, hypertension, and diabetes.

Our research shows that a significant difference between the frequency of the GSTM1null genotype in patient and control groups was identified. However, sub-group analysis found that the GSTM1-null genotype was statistically significant in men, but not in women, and in 
Caucasians, but not in Brown or Black groups. Furthermore, the GSTM1-null genotype was not related to any of the risk factors analyzed: cases in family, occupational exposure, smoking, hypertension, and diabetes.

\section{ACKNOWLEDGMENTS}

Research supported by Pontifícia Universidade Catóica de Goiás, Goiânia, Brazil (UCG/Prope/MGene). We are grateful to the Replicon staff and students who made this research possible.

\section{REFERENCES}

Anguria P, Ntuli S, Interewicz B and Carmichael T (2012). Traditional eye medication and pterygium occurrence in Limpopo Province. S. Afr. Med. J. 102: 687-690.

Asokan R, Venkatasubbu RS, Velumuri L, Lingam V, et al. (2012). Prevalence and associated factors for pterygium and pinguecula in a South Indian population. Ophthalmic Physiol. Opt. 32: 39-44.

Bolth HM and Thier R (2006). Relevance of the deletion polymorphisms of the glutathione S-tranferases GSTT1 and GSTM1 in pharmacology and toxicology. Curr. Drug Metab. 7: 613-628.

Cajucom-Uy H, Tong L, Wong TY, Tay WT, et al. (2010). The prevalence of and risk factors for pterygium in an urban Malay population: the Singapore Malay Eye Study (SiMES). Br. J. Ophthalmol. 94: 977-981.

Chui J, Di Girolamo N, Wakefield D and Coroneo MT (2008). The pathogenesis of pterygium: current concepts and their therapeutic implications. Ocul. Surf. 6: 24-43.

Di Girolamo N, Chui J, Coroneo MT and Wakefield D (2004). Pathogenesis of pterygia: role of cytokines, growth factors, and matrix metalloproteinases. Prog. Retin. Eye Res. 23: 195-228.

Evereklioğlu C, Er H, Doğanay S, Cekmen M, et al. (2003). Nitric oxide and lipid peroxidation are increased and associated with decreased antioxidant enzyme activities in patients with age-related macular degeneration. Doc. Opthalmol. 106: 129-136.

Frare A, Barbosa A, Costa I, Souza S, et al. (2013). GSTM1 and GSTT1 polymorphisms in endometriosis in women from Goiás, Brazil. Genet. Mol. Res. 12: 2764-2770.

Golu T, Mogoanta L, Streba CT, Pirici DN, et al. (2011). Pterygium: histological and immunohistochemical aspects. Rom. J. Morphol. Embryol. 52: 153-158.

Hayes JD, Flanagan JU and Jowsey IR (2005). Glutathione transferases. Annu. Rev. Pharmacol. Toxicol. 45: 51-88.

Jaworski CJ, Aryankalayil-John M, Campos MM, Fariss RN, et al. (2009). Expression analysis of human pterygium shows a predominance of conjunctival and limbal markers and genes associated with cell migration. Mol. Vis. 20: 2421-2434.

Luthra R, Nemesure BB, Wu SY, Xie SH, et al. (2001). Frequency and risk factors for pterygium in the Barbados Eye Study. Arch. Ophthalmol. 119: 1827-1832.

Ma K, Xu L, Jie Y and Jonas JB (2007). Prevalence of and factors associated with pterygium in adult Chinese: the Beijing Eye Study. Cornea 26: 1184-1186.

Meseret A, Bejiga A and Ayalew M (2008). Prevalence of Pterygium in a rural community of Meskan District, Southern Ethiopia. Ethiop. J. Health Dev. 22: 191-194.

Said T, Dutot M, Martin C, Beaudeux JL, et al. (2007). Cytoprotective effect against UV-induced DNA damage and oxidative stress: role of new biological UV filter. Eur. J. Pharm. Sci. 30: 203-210.

Scianni L, Filho CC, Lourenço GJ, Shinzato JY, et al. (2008). Polymorphisms GSTM1 and GSTT1 and sporadic breast cancer mammographic features. Rev. Assoc. Med. Bras. 54: 61-66.

Sherwin JC, Hewitt AW, Kearns LS, Griffiths LR, et al. (2013). The association between pterygium and conjunctival ultraviolet autofluorescence: the Norfolk Island Eye Study. Acta Ophthalmol. 91: 363-370.

Shiroma H, Higa A, Sawaguchi S, Iwase A, et al. (2009). Prevalence and risk factors of pterygium in a southwestern island of Japan: the Kumejima Study. Am. J. Ophthalmol. 148: 766-771.e1.

Solomon AS (2006). Pterygium. Br. J. Ophthalmol. 90: 665-666.

Suarez-Kurtz G, Vargens DD, Santoro AB, Hutz MH, et al (2014). Global pharmacogenomics: distribution of CYP $3 A 5$ polymorphisms and phenotypes in the Brazilian population. PLoS One 9: e83472.

Tsai YY, Lee H, Tseng SH, Cheng YW, et al. (2004). Null type of glutathione S-transferase M1 polymorphism is 
associated with early onset pterygium. Mol. Vis. 10: 458-461.

Tung JN, Wu HH, Chiang CC, Tsai YY, et al. (2010). An association between BPDE-like DNA adduct levels and CYP1A1 and GSTM1 polymorphisms in pterygium. Mol. Vis. 16: 623-629.

Viso E, Gude F and Rodríguez-Ares MT (2011). Prevalence of pinguecula and pterygium in a general population in Spain. Eye 25: 350-357.

Weiss JM, Goode EL, Ladiges WC and Ulrich CM (2005). Polymorphic variation in hOGG1 and risk of cancer: a review of the functional and epidemiologic literature. Mol. Carcinog. 42: 127-141.

West S and Muñoz B (2009). Prevalence of pterygium in Latinos: Proyecto VER. Br. J. Ophthalmol. 93: 1287-1290.

Young CH, Lo YL, Tsai YY, Shih TS, et al. (2010). CYP1Al gene polymorphisms as a risk factor for pterygium. Mol. Vis. 16: 1054-1058.

Zhao L, You QS, Xu L, Ma K, et al. (2013). 10-year incidence and associations of pterygium in adult Chinese: the Beijing Eye Study. Invest. Ophthalmol. Vis. Sci. 54: 1509-1514.

Zhong H, Cha X, Wei T, Lin X, et al. (2012). Prevalence of and risk factors for pterygium in rural adult Chinese populations of the Bai nationality in Dali: the Yunnan Minority Eye Study. Invest. Ophthalmol. Vis. Sci. 53: 6617-6621. 